\title{
Eurostudia
}

\section{Financer les cultes pour promouvoir le « vivre ensemble » et l’ordre public? La Belgique entre discours et réalités}

\section{Jean-François Husson}

Volume 13, numéro 1-2, 2018-2019

Cultures en contact, entre régulations et représentations

URI : https://id.erudit.org/iderudit/1064489ar

DOI : https://doi.org/10.7202/1064489ar

Aller au sommaire du numéro

Éditeur(s)

Le Centre canadien d'études allemandes et européennes

ISSN

1718-8946 (numérique)

Découvrir la revue

Citer cet article

Husson, J.-F. (2018). Financer les cultes pour promouvoir le " vivre ensemble " et l'ordre public? La Belgique entre discours et réalités. Eurostudia, 13(1-2), 71-92. https://doi.org/10.7202/1064489ar
Résumé de l'article

A l'origine, le régime belge de relations entre cultes et pouvoirs publics accordait une place centrale à l'ordre public et au contrôle social. Cette dimension de nature sécuritaire s'est estompée au fil du temps pour revenir en force, avec en point de mire le radicalisme islamique. En passant en revue les principaux traits du dispositif actuel - reconnaissance des cultes, organe représentatif, établissements cultuels, ministres des cultes -, l'auteur discute à la fois cette dimension d'ordre public ainsi que la contribution à la cohésion sociale. Il soulève un certain nombre de paradoxes rencontrés avant de conclure en discutant les avantages et les inconvénients du dispositif belge de cultes reconnus face à ces enjeux.
Tous droits réservés $@$ Le Centre canadien d'études allemandes et européennes, 2019
Ce document est protégé par la loi sur le droit d'auteur. L'utilisation des services d’Érudit (y compris la reproduction) est assujettie à sa politique d'utilisation que vous pouvez consulter en ligne.

https://apropos.erudit.org/fr/usagers/politique-dutilisation/ 
Financer les cultes pour promouvoir le «vivre ensemble » et l'ordre public? La Belgique entre discours et réalités

\author{
Jean-François Husson \\ UCLouvain, Université de Liège et Observatoire des Relations Administratives \\ entre les Cultes, la Laïcité organisée et l'Etat (ORACLE)
}

\title{
Résumé
}

A l'origine, le régime belge de relations entre cultes et pouvoirs publics accordait une place centrale à l'ordre public et au contrôle social. Cette dimension de nature sécuritaire s'est estompée au fil du temps pour revenir en force, avec en point de mire le radicalisme islamique. En passant en revue les principaux traits du dispositif actuel reconnaissance des cultes, organe représentatif, établissements cultuels, ministres des cultes -, l'auteur discute à la fois cette dimension d'ordre public ainsi que la contribution à la cohésion sociale. Il soulève un certain nombre de paradoxes rencontrés avant de conclure en discutant les avantages et les inconvénients du dispositif belge de cultes reconnus face à ces enjeux.

\section{Abstract}

Originally, the Belgian system of relations between religious communities and public authorities gave a central place to public order and social control. This dimension has faded over time to then make a strong comeback, with a focus on Islamic radicalism. By reviewing the main features of the current system - recognition of communities, representative body, religious institutions, ministers of religion - the author discusses both this dimension of public order and 
the contribution to social cohesion. He raises a number of paradoxes encountered before concluding by discussing the advantages and disadvantages of the Belgian system of recognized religions in facing these issues.

Un argument $\mathrm{du}$ discours politique en faveur $\mathrm{du}$ système de reconnaissance et de financement des cultes - en particulier en Belgique - est que ces dispositifs contribuent au «vivre ensemble» et à l'ordre public. La présente contribution propose de revenir aux origines du dispositif belge puis de présenter une analyse critique de ses principales composantes - la reconnaissance des cultes et celle d'un organe représentatif, les ministres du culte et les communautés cultuelles locales - avant d'aborder quelques réflexions transversales ${ }^{1}$.

\section{Aux origines de l'actuel régime de cultes reconnus}

Le régime existant en Belgique est un système de cultes et organisations philosophiques reconnus, dont les instruments sont restés quasiment inchangés depuis avant l'indépendance. En effet, les territoires de l'actuelle Belgique ayant été annexés par la France en 1795, le Concordat de 1801 et les législations en découlant y sont appliqués, mettant en place un dispositif basé sur la civilisation paroissiale : les fidèles se retrouvent au lieu de culte (dont l'entretien est à charge de la commune) sous la guidance du ministre du culte (payé par l'État), le coût

\footnotetext{
${ }^{1}$ Par manque de place, d'autres éléments liés à la reconnaissance ne seront pas abordés, qu'il s'agisse des aumôniers («conseillers islamiques»), des émissions cultuelles sur les chaines publiques, ou de l'organisation du cours de religion islamique. Voir par exemple Husson (2012), Husson et Mandin (2014) ou encore http://www.centre-craig.org/categorie/le-financement-de-l-islam-en-belgique.html pour d'autres sources et plus d'information.
} 
$\mathrm{du}$ fonctionnement $\mathrm{du}$ culte étant pris en charge par la commune en cas d'insuffisance des moyens de la communauté paroissiale.

La Révolution belge de 1830 débouche en 1831 sur une Constitution particulièrement libérale pour l'époque. Elle reflète le compromis «à la belge » survenu entre les deux courants politiques d'alors: les catholiques (conservateurs), qui obtiennent le maintien du financement des cultes et la liberté d'enseignement, et les libéraux, qui engrangent la liberté de conscience et la liberté de la presse. Le catholicisme - alors religion dominante - n'est pas établi en religion d'État et aucun Concordat n'est conclu bien qu'une série de ses instruments - notamment financiers - soient maintenus ${ }^{2}$, de même que la reconnaissance des cultes protestant et israélite. Ces divers équilibres annonçaient un régime de consociation (Lijphart 1981).

Le système belge apparaît à l'époque paradoxal car il organise un financement non négligeable sans l'accompagner de mesure de police des cultes telles celles instaurées par le Concordat. Toutefois, il s'inscrit dans l'optique d'une «utilité sociale» visant non un rôle caritatif des cultes, mais leur contribution à garantir l'ordre social, à renforcer la moralité publique, et à faire respecter les lois, la propriété, les biens et les personnes (de Coorebyter 2005 : 98-99). Tout au long du XIXe siècle, l'Église fut perçue comme un instrument de l'ordre public, Lebeau ${ }^{3}$ écrivant ainsi dans les années 1850 : «un curé de village vaut mieux que cent gendarmes pour le maintien de l'ordre » (cité par Keunings 2009: 41). Dans les décennies suivantes, d'autres voyaient l'enseignement catholique et le cours de religion comme un rempart contre le socialisme.

\footnotetext{
${ }^{2}$ Cf. Articles organiques et plusieurs décrets, dont le décret impérial du 30 décembre 1809 sur les fabriques des églises.

${ }^{3}$ Joseph Lebeau, 1794-1865, homme politique libéral, plusieurs fois ministre et chef de l'exécutif.
} 
À l'époque, la «paix des religions» et le dialogue interconvictionnel n'étaient guère à l'agenda. Il n'en était nul besoin : le poids de l'Église catholique (plus de $99 \%$ de la population) ${ }^{4}$ suffisait à une certaine régulation de la société, realpolitik oblige ${ }^{5}$. Cela n'empêchait pas des conflits entre catholiques et libéraux, notamment en matière scolaire, amenant le monde catholique à constituer son réseau d'enseignement, amorçant un "vivre côte à côte", typique de la segmentation traditionnelle de la société belge qu'incarnera par la suite la « pilarisation $»^{6}$.

Cette dimension $\mathrm{d}^{\prime}$ « ordre public » ayant perdu de l'importance au fil $\mathrm{du}$ temps, l'utilité sociale a ensuite été perçue comme une assistance morale et religieuse à apporter à la population. C'est d'ailleurs dans cette perspective que les reconnaissances du culte islamique et de la «laïcité organisée » furent proposées pour la première fois en 1970, mais aussi, plus récemment, que des commissions d'experts ont abordé la question du statut des ministres des cultes (Christians et al. 2010; Rigaux et al. 2006) ou de leur formation (Husson et Mandin 2014).

Depuis plusieurs années (Torfs 2005 : 21), les considérations d'ordre public sont à nouveau inscrites sur l'agenda politique au point de redevenir une dimension intrinsèque d'une «politique des cultes ». En Belgique, l'attention a davantage été portée sur l'islam depuis la fin des années 70 et surtout depuis les émeutes survenues à Forest en 1991, au cours desquelles il a été demandé à des représentants du culte islamique d'appeler au retour au calme. Depuis 2015, la majorité des dossiers relatifs au culte islamique ont été traités à l'aune de la lutte

\footnotetext{
${ }^{4}$ D'après le recensement de 1846 (Rigaux et al. 2006).

${ }^{5}$ Pour rappel, le premier Roi des Belges, Léopold Ier, était luthérien, mais s'appuyait fortement sur l'Église catholique.

${ }^{6}$ Le CRISP définit un pilier comme un « ensemble d'organisations qui ont une idéologie commune et qui veillent à son influence dans l'organisation de la société » (http://www.vocabulairepolitique.be/pilier, consulté le 17 mai 2016). On parle notamment du « pilier chrétien ».
} 
contre le radicalisme, la « qualité du service » aux fidèles passant au second plan, de même que d'autres dossiers (reconnaissance du bouddhisme, mouvements religieux contestés, etc.). Ce retour à l'agenda s'est traduit par des mesures relatives aux cadres religieux (e.g. formation, "screening ") et des mesures de «police $»^{7}$. Remarquons cependant que d'autres situations ont fait l'objet d'une attention des services de sécurité, bien que cela n'ait guère été médiatisé : il s'agit notamment des communautés orthodoxes dépendant des Églises nationales de pays du Pacte de Varsovie durant la guerre froide et de communautés orthodoxes serbes durant les guerres en ex-Yougoslavie ${ }^{8}$. Quant à d'autres communautés convictionnelles, non reconnues, elles ont pu faire l'objet d'un suivi attentif $d u$ Centre d'Information et d'Avis sur les Organisations sectaires nuisibles" notamment certaines communautés évangéliques et pentecôtistes. Ces derniers exemples illustrent d'ailleurs une double évolution à l'œuvre depuis le début de ce siècle: d'une part, il existe un certain nombre de communautés qui ne s'affilient pas ou ne se sentent pas représentées par l'organe représentatif de leur courant religieux ${ }^{10}$ et, d'autre part, la délimitation entre « culte » et « secte » est devenue plus floue ${ }^{11}$.

Apportons ici une première appréciation. Beckford et Richardson (2007) distinguent trois sphères de régulation dans le champ religieux : l'autorégulation du religieux, la régulation (de la société) par le religieux et la régulation du

\footnotetext{
${ }^{7}$ Ce terme est à prendre d'une manière très générale, allant de l'exercice de la tutelle administrative à d'autres mesures de police administrative jusqu'à des inspections de lieux de culte par les services de police.

${ }^{8}$ Ces situations n'ont pas fait l'objet de publications à notre connaissance.

${ }_{9}^{9}$ Organe officiel, mais indépendant, institué au niveau fédéral; www.ciaosn.be.

${ }^{10}$ Un nombre substantiel de communautés « évangéliques » (au sens large) ne sont pas affiliées au Synode Fédéral, branche évangélique du culte protestant-évangélique reconnu.

11 Aucune disposition légale ne vise les sectes en tant que telles en Belgique; a contrario, les comportements sectaires nuisibles (c.-à-d. des activités criminelles ou délictueuses menées sous une couverture religieuse ou philosophique) le sont. Certains groupements évangéliques et au moins un groupement bouddhique, le cas échéant considérés comme "secte » devant faire l'objet d'un suivi dans d'autres pays, peuvent apparaître comme des cas limites.
} 
religieux (principalement par l'État). La démarche belge, pragmatique, peut être succinctement résumée en disant que, à défaut d'autorégulation du religieux ${ }^{12}$, le producteur de politique publique va tenter de réguler le religieux afin qu'à son tour il régule sa communauté de foi et contribue ainsi à la régulation de la société dans son ensemble, non plus dans une perspective de norme morale, comme sous le Consulat et l'Empire (Portalis 1845), mais de cohésion sociale ${ }^{13}$.

\section{Les communautés convictionnelles reconnues}

L'art. 181 de la Constitution, sur la base duquel sont payés les traitements et pensions des ministres des cultes, a été élargi en 1993 aux organisations fournissant une assistance morale non confessionnelle, d'où l'utilisation officieuse du terme générique de « communautés convictionnelles ».

Aux cultes catholique, protestant et israélite des origines, s'est ajouté le culte anglican (en diverses étapes, avec reconnaissance formelle en 1870) puis, un siècle plus tard, les cultes islamique (1974) et orthodoxe (1985). Le courant évangélique a été implicitement reconnu par un élargissement du culte protestant au culte protestant-évangélique au début des années 2000. Quant aux organisations qui offrent une assistance morale selon une conception philosophique non confessionnelle, le courant humaniste incarné par la «laïcité organisée » a été reconnu en plusieurs étapes entre 1981 et 2002 (Husson et Sägesser 2002) tandis que le bouddhisme est dans une phase préalable à la

\footnotetext{
${ }^{12}$ Contrairement au culte islamique, les autres cultes et organisations philosophiques ont pu parvenir à des arrangements visant à déterminer un organe représentatif et un certain nombre de règles au sein du courant religieux concerné, même si cela ne s'est pas toujours fait sans difficultés (comme dans le cas du regroupement protestant-évangélique par exemple).

${ }^{13}$ La cohésion sociale "désigne la capacité d'une société à garantir le bien-être de tous ses membres, en réduisant les disparités au minimum et en évitant les polarisations. Une société cohésive est une communauté solidaire d'individus libres poursuivant ces objectifs communs par des moyens démocratiques » (Conseil de l'Europe $2008: 10$ ).
} 
reconnaissance officielle depuis 2006, l'adoption d'une législation organique ayant souffert de divers reports ${ }^{14}$.

S'il n'y a pas de texte légal définissant les critères de reconnaissance d'un culte ou d'une organisation non confessionnelle - la question ne s'est d'ailleurs pas réellement posée avant la fin du XXe siècle - les réponses ministérielles aux questions parlementaires ont régulièrement mentionné cinq critères: être organisé, être présent dans le pays depuis un certain temps, présenter un intérêt social, compter un certain nombre d'adhérents et ne pas avoir d'activités contraires à l'ordre public. Dernière procédure en date, la demande de reconnaissance de l'Union Bouddhique Belge a été examinée sur la base de ces critères.

Enfin, contrairement à d'autres pays dont la France ${ }^{15}$, si l'arrivée de l'islam sur le «marché convictionnel» belge n'a pas été à l'origine de l'ouverture du débat sur le financement public des cultes ${ }^{16}$, elle a amené les pouvoirs publics à reconsidérer les questions de «la paix des religions», du dialogue interconvictionnel et de l'ordre public, et à appréhender différemment la reconnaissance et le financement des communautés cultuelles, notamment en termes de reconnaissance symbolique ${ }^{17}$.

Une deuxième conclusion est que le dispositif belge apparaît ouvert et a pu faire preuve d'une capacité d'adaptation, tant au XIXe siècle (culte anglican) qu'à la fin du $X X^{\mathrm{e}}$ siècle. Certes, dans divers cas, des difficultés de mise en œuvre sont

\footnotetext{
${ }_{14}$ Notamment l'impossibilité de conclure avant les élections fédérales de 2014, les attentats islamistes de 2018, la crise politique débouchant sur un gouvernement minoritaire d'affaires courantes en 2018-19.

${ }^{15}$ Voir notamment : Haut Conseil à l'intégration (2000) et Machelon (2006).

${ }^{16}$ Le débat découle davantage d'une demande de milieux laïques non pas de supprimer, mais de répartir autrement les moyens concernés, notamment via une correction en leur faveur.

${ }_{17}$ Dans la lignée des travaux de Charles Taylor ou Axel Honneth.
} 
apparues, mais une part de celles-ci résultent de circonstances politiques externes ou de problèmes propres à la communauté convictionnelle concernée.

\section{Les organes représentatifs}

Qui dit culte reconnu, dit nécessité pour l'autorité civile de disposer d'un interlocuteur, et donc d'un organe représentatif. Compte tenu des arrangements politico-administratifs hérités de l'époque française, la question s'est posée véritablement pour la première fois dans les années 1870 pour le culte anglican : un comité anglican, dont la composition était déterminée par le gouvernement belge, fut institué, compte tenu du fait que l'Église d'Angleterre dépendait du souverain et du gouvernement britannique (Sägesser 2013 : 286-291; Wynants 1986: 161-162). Cela ne manque pas de renvoyer au risque d'une influence étrangère au travers de certains cultes ${ }^{18}$.

L'option prise de ne reconnaître qu'un organe représentatif par grand courant religieux a de facto été questionnée lorsque la question s'est reposée à partir des années 60-70. Nouvellement reconnus ou devant intégrer de nouveaux courants, les cultes minoritaires ont finalement $\mathrm{pu}$ s'accorder sur une forme d'organe représentatif ${ }^{19}$, ce qui n'a pas empêché des tensions voire des blocages (cas du culte protestant-évangélique) ou de devoir faire preuve d'un certain pragmatisme (cultes israélite et orthodoxe). Cela s'est toutefois avéré plus compliqué dans le cas du culte islamique. Si le gouvernement adopta en 1978 un arrêté royal désignant comme organe représentatif le Centre Islamique et Culturel de Belgique - lié à l'Arabie Saoudite et la Ligue islamique mondiale et auquel la gestion de la Grande mosquée avait été confiée - , cet arrêté ne fut finalement pas mis en œuvre et, devant des contestations, d'autres pistes furent

\footnotetext{
${ }^{18}$ Crainte présente depuis la Réforme (notamment en France à l'encontre des protestants et en Angleterre à l'encontre des catholiques); cette question a retrouvé une actualité avec le culte islamique et, d'une manière moins médiatisée, le culte orthodoxe.

${ }^{19} \mathrm{Il}$ en va de mêmes pour la « laïcité organisée » (humanistes) et l’Union Bouddhique belge.
} 
explorées pour aboutir à l'Exécutif des Musulmans de Belgique (EMB) ${ }^{20}$. Depuis, quel que soit le mode de désignation utilisé (élections en 1998 et 2005, désignation par les représentants de mosquées en 2014), le caractère représentatif de l'EMB a été contesté, en interne comme en externe, et son poids reste très faible par rapport aux «organisations coupoles», c.-à-d. les fédérations de mosquées telle le Diyanet lié à l'État turc ${ }^{21}$. Et quant à sa mission de gestion du temporel du culte, le mode de désignation retenu en 2014 donnant une majorité de votants issus de mosquées non reconnues ou ne souhaitant pas être reconnues, a créé une situation quelque peu paradoxale.

$\mathrm{Au}$ niveau de ces organes représentatifs, la dimension sécuritaire est apparue dans les années 1990. Ainsi, les membres élus à la suite des élections de l'EMB de 1998 firent l'objet d'un «avis » de la Sûreté de l'État et ceux issus de celles de 2005 d'un «screening », ou vérification de sécurité, sur la base de la loi du 3 mai 200522. L'éviction de membres élus de l'Exécutif sur ces bases ayant généré de vives tensions, la ministre ayant les cultes dans ses attributions s'est abstenue d'y procéder après les élections de 2014. Cet arrêté royal de désignation des membres de l'EMB faisant cependant l'objet de recours, le ministre lui ayant succédé a pris les devants en expurgeant le processus de désignation de l'EMB de toute intervention de l'État ${ }^{23}$ : dorénavant, celui-ci n'est plus qu'informé de l'identité des personnes clés et la procédure de «screening» est de facto supprimée.

\footnotetext{
${ }^{20}$ Pour l'historique, voir Husson et Mandin (2014) ou Sägesser et Torrekens (2008).

${ }^{21}$ Ce problème est rencontré dans de nombreux pays européens, quel que soit le régime de relations Églises-Etats (France, Grande-Bretagne, Allemagne,...).

${ }^{22}$ Les dispositions de cette loi s'appliquent aux membres des organes représentatifs des cultes, désignés par arrêté royal et qui ne sont pas des ministres des cultes, ainsi qu'à l'ensemble des aumôniers et conseillers moraux en milieu pénitentiaire. De facto, seul l'EMB était alors concerné.

23 Arrêté royal du 15 février 2016 portant reconnaissance de l'Exécutif des Musulmans de Belgique, modifié par un arrêté royal du 18 avril 2017.
} 
Posons ici plusieurs constats. La reconnaissance d'un seul organe représentatif par grand courant religieux amène les diverses composantes de celui-ci à devoir négocier entre elles - sorte de version interne de la " paix des religions » évoquée par ailleurs - et facilite la gestion de dossiers transversaux comme les aumôneries ou les cours de religion. A contrario, cela débouche dans certains cas sur des situations de conflits, voire de blocages, liées à des lignes idéologiques auxquelles peuvent s'ajouter parfois des clivages liés à certains intérêts nationaux.

Ensuite, lorsque la légitimité d'un organe représentatif est contestée au sein de ses instances et au sein de sa communauté, peut émerger une situation qui attise les tensions au lieu de contribuer à la «cohésion sociale » 24 , l'intervention éventuelle des pouvoirs publics pour contribuer à la recherche de solutions pouvant apparaître (ou être présentée par certains acteurs) comme une tentative d'ingérence.

Enfin, concernant l'EMB, alors que la dimension sécuritaire est considérée comme prioritaire, l'État a renoncé à ses possibilités de contrôle, compte tenu des tensions que l'application de celles-ci générait. Cela met fin à tout traitement différencié du culte islamique en la matière, mais l'existence du «screening » (bien que n'étant plus d'actualité) reste un argument régulièrement avancé pour dénoncer un traitement discriminatoire du culte islamique.

\section{Les communautés cultuelles locales}

Les communautés locales d'un culte reconnu par l'Autorité fédérale peuvent être reconnues par les Régions, compétentes en la matière depuis le $1^{\mathrm{er}}$

\footnotetext{
${ }^{24}$ Peut se poser également la question de l'efficacité des actions financées par les pouvoirs publics au niveau de l'organe représentatif en pareil cas.
} 
janvier $2002^{25}$. Chacune a depuis adopté ses propres critères de reconnaissance, lesquels prennent en compte la dimension d'ordre public, tant pour l'institution que pour les personnes qui $1^{\prime}$ administrent ${ }^{26}$. De plus, ces reconnaissances s'opèrent dans le cadre d'un Accord de coopération avec l'autorité fédérale : si cette dernière remet un avis négatif pour des raisons relatives à la sécurité de l'État ou à l'ordre public, la reconnaissance de la communauté locale est suspendue.

Une telle reconnaissance entraîne la constitution d'un établissement public chargé des seules activités cultuelles, pour lequel les pouvoirs locaux (communes, provinces ou Région de Bruxelles-Capitale selon les cas) pallient le déficit et pourvoient aux grosses réparations à l'édifice du culte, outre la mise à disposition d'un logement au ministre du culte ${ }^{27}$. Pour les communautés qui fonctionnaient sur un mode associatif et combinaient activités sociales, culturelles et cultuelles, la reconnaissance implique une scission entre leurs activités cultuelles d'une part et socioculturelles d'autre part. Soulignons également qu'aucun financement n'est prévu pour les activités interconvictionnelles, sauf le cas échéant des subsides facultatifs d'une ampleur limitée.

\footnotetext{
${ }^{25}$ La Région wallonne a transféré l'exercice de cette compétence à la Communauté germanophone sur le territoire de cette dernière.

${ }^{26}$ En Flandre, la liste des critères est particulièrement complète : viabilité financière (plan financier); intérêt social (nombre estimé de fidèles; intégration dans la vie locale : utilisation du néerlandais dans les contacts avec les fidèles et tiers; contacts avec les autorités civiles, etc.); application de la législation linguistique; respect des dispositions en matière d'inburgering; engagement de ne pas aller à l'encontre de la Constitution et de la Convention européenne des droits de l'homme. En Wallonie, les critères sont la conformité du lieu de culte, l'existence d'une structure juridique adaptée et « l'absence de violation, par les membres des organes de gestion de l'établissement cultuel dont le ou les Ministres du culte, de la Constitution, la Convention de sauvegarde des droits de l'homme et des libertés fondamentales et l'ensemble des législations existantes, ainsi que la démonstration de leur capacité de gestion administrative et financière ». La Région de Bruxelles-Capitale prépare une nouvelle législation tandis que la Communauté germanophone, qui ne compte que des établissements reconnus catholiques et protestants, n'a pas fixé de tels critères pour l'instant.

${ }^{27} \mathrm{Ou}$ le versement d'une indemnité compensatoire.
} 
La volonté politique est d'identifier les communautés cultuelles locales et que, au sein des cultes reconnus (et en particulier du culte islamique) un maximum d'entre elles soient reconnues. La perception du politique est en effet qu'une communauté reconnue pourra être «régulée» par son organe représentatif et que les dispositifs de tutelle sur certains de ses actes civils et financiers permettront une certaine transparence, voire un certain contrôle.

À cet égard, la situation apparaît paradoxale à trois égards. D'abord, a contrario de la volonté affirmée d'encourager la reconnaissance, on peut constater qu'en Région wallonne, plus aucune demande n'a été traitée depuis la vague (massive, il est vrai) de reconnaissance de mosquées en 2007. Ensuite, les conditions de reconnaissance sont devenues de plus en plus strictes, comme l'illustre le décret wallon de 2017. Enfin, hormis la prise en charge du traitement du ministre du culte - mais celle-ci n'intéresse pas toutes les communautés comme évoqué par la suite - l'intérêt financier d'une reconnaissance peut apparaître limité, en regard de ce qui est perçu comme les contraintes y associées (tutelle, loi sur les marchés publics, etc.). C'est là le premier paradoxe : une voie est préconisée, mais rendue plus ardue.

Quant à la transparence et au contrôle, force est de constater que nombre d'établissements publics islamiques ont des difficultés à suivre la réglementation administrative, malgré un soutien administratif externe et une certaine mansuétude de l'administration. Certains flux financiers à destination d'établissements publics ont également posé question ${ }^{28}$. Enfin, il a été constaté que nombre d'associations organisant les activités socioculturelles, liées aux établissements reconnus, n'étaient pas en règle vis-à-vis de leur législation

28 Cf. www.lecho.be/economie-politique/belgique-general/Plus-de-transparence-pour-les-financementsetrangers-des-ASBL/10044282, et www.lecho.be/economie-politique/belgique-general/Commentemanciper-1-islam-de-Belgique-des-tutelles-etrangeres/9938732, consultés le 14/01/2019. 
organique $^{29}$. Le second paradoxe est donc qu'en dépit des efforts déployés, cet objectif de transparence et de contrôle n'est que partiellement rencontré.

Troisième paradoxe, l'organisation de conférences ou d'autres activités à connotation cultuelle par des associations a parfois été signalée, au grand dam de l'EMB qui se trouve ainsi empêché de jouer son rôle de «»régulateur » qui ne peut porter que sur les établissements publics et, ce, dans une mesure limitée. La démarcation entre cultuel et culturel apparaît ainsi des plus ténues.

\section{Les ministres des cultes}

Venons-en aux ministres du culte, dont la prise en charge des traitements et pensions est prévue par l'art. 181 de la Constitution. Chaque culte se voit attribuer un cadre basé sur le nombre de postes attribués aux communautés locales $^{30}$ et de postes d'encadrement et administratifs pour son organe représentatif ${ }^{31}$. Les ministres du culte ont un statut sui generis (Beumier 2006; Rigaux et al. 2006) et leurs traitements sont fixés par la loi ${ }^{32}$. À noter que depuis plusieurs années, le formulaire à remplir pour la prise en charge du traitement du ministre du culte par l'État prévoit un engagement de respecter les lois et dispositions légales applicables en Belgique et de ne pas être rémunéré par une institution étrangère.

\footnotetext{
${ }^{29}$ Constat émanant du Plan Canal (une vaste opération de vérification de certaines personnes physiques et morales dans plusieurs communes bruxelloises situées à proximité du Canal) et portant sur certaines associations islamiques et évangéliques; voir aussi p. ex. www.sudinfo.be/id82265/article/2018-1026/largent-cache-des-asbl-des-mosquees-selon-la-loi-elles-sont-tenues-de-deposer (consulté le 14/01/2019).

${ }^{30}$ Les communautés locales non reconnues d'un culte reconnu ne peuvent disposer d'un ministre du culte dont le traitement est pris en charge par les pouvoirs publics.

31 À l'exception du culte anglican. Dans le cas du culte islamique, les premiers postes pris en charge dès 2006 étaient d'ailleurs 4 postes à l’EMB (secrétaire général, secrétaire général adjoint, comptable et traducteur).

${ }^{32}$ Loi du 2 août 1974 relative aux traitements des titulaires de certaines fonctions publiques, des ministres du culte et des délégués du Conseil central laïque.
} 
À l'exception du pan évangélique du culte protestant-évangélique et du culte islamique, la quasi-totalité des ministres des cultes sont ainsi rémunérés par l'État bien qu'étant désignés en toute indépendance par leur organe représentatif. Les autres sont à charge de leur communauté ou financés par un État étranger. La situation est problématique pour les mosquées turques reconnues et affiliées au Diyanet (soit la majorité des mosquées reconnues) qui refusent un imam pris en charge par l'État belge et disposent d'un imam envoyé par le Diyanet turc. Cette situation, tolérée jusqu'à présent, pourrait être remise en cause par les pouvoirs publics souhaitant s'affranchir de ce qui est perçu comme une ingérence étrangère, l'imam étant de plus membre de droit du comité de gestion de la mosquée reconnue, laquelle est un établissement public. Il est à prévoir qu'en pareil cas les mosquées turques concernées sortent du cadre de la reconnaissance pour revenir à un simple statut associatif, renforçant ainsi le premier paradoxe cité dans la section précédente.

Une attente des pouvoirs publics à l'égard des imams a été qu'ils puissent calmer les esprits dans des moments de tensions. «Un imam vaut cent gendarmes » s'est en quelque sorte substitué à " un curé vaut cent gendarmes » évoqué précédemment, comme en témoignent les mesures concernant la formation des cadres musulmans dans les divers plans et initiatives antiradicalisation $^{33}$. Comme dans d'autres pays européens (Husson 2007, 2018; Husson et Dury 2006; Husson et Mandin 2014), la volonté du politique a ainsi été de s'assurer de la qualité de la formation des imams pour prodiguer un accompagnement moral et religieux de qualité à destination des fidèles, mais aussi pour pouvoir contrer les discours radicaux; cette formation devait comporter un volet théologique, mais, également, une connaissance de la société

${ }^{33}$ Voir à titre illustratif RTBF (2016) ou Blogie (2015). 
d'accueil pour les ministres du culte d'origine étrangère ${ }^{34}$. Les connaissances théologiques des imams postulant dans une mosquée reconnue sont évaluées par le Conseil des théologiens, qui peut considérer qu'un candidat n'a pas le niveau requis ${ }^{35}$. A contrario, on remarquera que rien n'est prévu pour d'autres courants religieux où la qualité de la formation des ministres du culte est très inégale, en particulier pour le courant évangélique (cf. Rigaux et al. 2006 : 177).

Enfin, rappelons que le Code pénal contient toujours une disposition sanctionnant « les ministres d'un culte qui dans l'exercice de leur ministère, par des discours prononcés en assemblée publique, auront directement attaqué le gouvernement, une loi, un arrêté royal ou tout autre acte de l'autorité publique ». Cette disposition remontant à l'époque concordataire a toutefois été rappelée par la ministre de la Justice aux organes représentatifs en 2006.

\section{La «paix des religions"}

Quant à la «paix des religions», longtemps entendue comme une « coexistence pacifique », elle a au fil du temps pris une dimension de négociation des intérêts (position commune en matière d'aumôniers de prisons par exemple) ou des valeurs. Cette dernière vision semble confirmée par la création, entamée dès 2010 en Flandre, d'une plateforme interconvictionnelle destinée à débattre d'enjeux de société et l'annonce récente d'une initiative similaire au niveau fédéral, à la suite des attentats de Bruxelles du 22 mars 2016. Cependant, aucun dispositif de financement ne vise cet objectif; l'optique du « côte à côte » - dans la droite ligne de la «pilarisation» belge - continue à prédominer dans l'organisation du financement (Husson 2017). Le dialogue interconvictionnel se situe donc d'une part au niveau des élites (organes représentatifs) et d'autre part

\footnotetext{
${ }^{34}$ En Flandre, la participation de l'imam au parcours d'intégration civique (inburgeringsbeleid) est un des éléments pris en compte dans le dossier de reconnaissance d'une mosquée.

${ }^{35}$ La composition de ce Conseil, assez discret, est également contestée, d'aucuns le considérant comme trop conservateur et peu ouvert à d'autres tendances.
} 
au niveau local, en fonction d'initiatives non concertées. Cela peut a priori sembler paradoxal alors que le discours politique promeut le «vivre ensemble » dans le cadre de la cohésion sociale, laquelle est d'ailleurs approchée différemment selon les Régions. Cette absence de cadre institutionnel n'a cependant pas empêché les responsables des cultes et des organisations philosophiques non confessionnelles de se mobiliser à plusieurs reprises à l'occasion de manifestations d'hommages ou de solidarité à la suite des attentats en France et en Belgique.

\section{Remarques conclusives}

La Belgique reconnait et finance les communautés convictionnelles au titre de leur rôle (de régulation) dans une perspective $\mathrm{d}^{\prime}$ «utilité sociale». Cette dernière est passée d'une contribution à l'ordre public et à la défense de l'ordre établi à une régulation d'une communauté de foi participant d'une contribution à la cohésion sociale. De ce fait, la dimension sécuritaire, très importante à l'origine, a ensuite été ramenée à des considérations ponctuelles pendant une bonne partie $\mathrm{du} X X^{\mathrm{e}}$ siècle avant de se retrouver quasiment au centre des politiques concernant les communautés convictionnelles, avec en point de mire le radicalisme islamique. Quant à la dimension interconvictionnelle, les divers acteurs politiques, convictionnels et sociaux ont encore du mal à véritablement la définir et la traduire en instruments : c'est à peine caricaturer que de dire que le «vivre ensemble » est aussi présent dans le discours politique qu'il est absent des canaux de financement, et ce alors que nombre d'indicateurs sont préoccupants ${ }^{36}$.

À son crédit, le dispositif belge a pu facilement s'accommoder de nouveaux acteurs convictionnels, dont l'islam et le courant humaniste (Husson 2014), témoignant d'une grande ouverture et d'un certain pragmatisme. Constatons

\footnotetext{
36 Voir par exemple les rapports d'Unia (anciennement centre fédéral pour l'égalité des chances): www.unia.be.
} 
toutefois que les interventions, parfois maladroites, des pouvoirs publics afin de permettre au culte islamique de disposer d'un organe représentatif à même d'actionner tous les instruments liés à la reconnaissance (Husson et Mandin 2014; Sägesser et Torrekens 2008) ont régulièrement été dénoncées par certains acteurs comme des ingérences, contribuant à délégitimer ledit organe, y compris après le retrait des dispositions incriminées, tel le «screening ». Le cas du culte islamique illustre une faiblesse du système: il ne peut fonctionner que si chaque communauté convictionnelle reconnue peut constituer un organe qui est représentatif et qui fonctionne. Le CACPE, organe du culte protestantévangélique, a connu certains blocages à la suite de conflits entre ses deux composantes, mais sa représentativité ne peut être mise en doute. Du côté de $l^{\prime} E M B$, sa représentativité a constamment été contestée, ce qui n'a toutefois pas empêché un certain fonctionnement. Si l'option politique d'un organe représentatif unique pour chaque grande communauté convictionnelle a $\mathrm{d}$ 'incontestables avantages (notamment pour les dossiers transversaux que sont les cours de religion dans l'enseignement public ou les aumôneries), elle a aussi des limites qui découlent notamment des négociations d'intérêt et valorielles internes à chaque communauté convictionnelle.

Quid de la reconnaissance des communautés locales? Elle entraîne également un certain nombre d'incompréhensions. Les contraintes réglementaires et administratives apparaissant à d'aucuns comme lourdes ou comme une mesure supplémentaire de contrôle à l'égard des musulmans, alors qu'elles sont d'application pour l'ensemble des cultes reconnus. Nous avons également soulevé les paradoxes autour de ces reconnaissances. Le premier d'entre eux est que les pouvoirs publics souhaitent les encourager alors que parallèlement les critères et modalités pratiques se font plus stricts, les incitatifs financiers sont limités et que, dans le cas des mosquées turques du Diyanet, se pose la question des imams envoyés par la Turquie. Un second est que ces 
reconnaissances sont encouragées parce que perçues comme un moyen d'avoir un regard sur les activités des lieux de culte et, surtout, sur leur gestion administrative, mais, en pratique, ce regard est très limité.

Les initiatives publiques en matière de formation des cadres religieux sont également diversement accueillies : appelées de leurs vœux par d'aucuns, elles sont rejetées sans ménagement par d'autres parties de la communauté musulmane qui y voient une ingérence. En matière d'évaluation d'une politique publique, ces initiatives peuvent donc être questionnées en matière de légitimité, d'efficience et d'efficacité (Husson et Mandin 2014). Faut-il dès lors vouer aux gémonies le système de cultes reconnus?

Si les instances reconnues du culte islamique (EMB et mosquées reconnues) ne sont pas, par ce seul fait, immunisées d'une influence émanant de courants radicaux, les situations problématiques relevées par les médias ou la Justice relèvent davantage de mosquées non reconnues. De même, les phénomènes de radicalisation ne se déroulent généralement pas au départ des mosquées $-a$ fortiori reconnues - mais via internet, des réseaux interpersonnels et des imams autoproclamés et la problématique mérite d'être appréhendée plus largement, en identifiant par exemple les raisons des frustrations exprimées par les jeunes d'origine marocaine afin de chercher les solutions à y apporter (Torrekens et Adam 2015).

Concernant les relations avec les pouvoirs publics, l'avantage d'un régime de reconnaissance est que les principaux interlocuteurs sont connus ${ }^{37}$. A contrario, une «neutralité d'abstention » ou «neutralité par le vide » pourrait se traduire par une absence de contacts avec les cultes et communautés philosophiques, avec le risque que les pouvoirs publics souffrent d'un déficit de

\footnotetext{
${ }^{37}$ Avec un léger bémol pour les communautés cultuelles locales reconnues sur une base provinciale (c.-àd. les cultes islamique et orthodoxe).
} 
connaissance qui pourrait les amener à des «confusions, décisions au jugé, postures défensives » (Hermon-Belot et Fath 2005), comme l'illustre par exemple le choix d'un interlocuteur inadéquat par le gouvernement danois au moment de la crise liée aux dessins du Prophète (Favret-Saada 2007).

Concluons avec deux dernières remarques. D'abord, nous pensons qu'un dispositif de cultes reconnus peut présenter un intérêt pour une politique de cohésion sociale mais, pour cela, il faudrait que les objectifs de cette politique soient davantage définis, sous peine pour les instruments concernés de rencontrer des problèmes de légitimité, d'efficience ou d'efficacité, comme nous l'avons déjà évoqué au sujet de la formation des cadres religieux.

Ensuite, soulignons que les pays européens, en dépit d'histoires nationales propres ayant établi des systèmes de relations Églises-États différenciés, se trouvent confrontés à des problématiques communes - telle la formation des cadres musulmans - et s'orientent vers des solutions convergentes. La question de l'émergence éventuelle d'un certain modèle européen de relations ÉglisesÉtats ou de laïcité européenne (Milot et al. 2010; Torfs 2009; Willaime 2008, 2009) peut alors être soulevée. Il s'agit là d'un autre et vaste débat que nous nous bornerons ici à évoquer.

\section{Bibliographie}

Beckford, J. A. et J. T. Richardson. (2007). « Religion and Regulation ». Dans J. A. Beckford et J. Demerath III (dir.), The SAGE Handbook of the Sociology of Religion. Londres, SAGE, p. 396-418. 
Beumier, M. (2006). "Le statut social des ministres des cultes et des délégués laïques ». Courrier hebdomadaire du Centre de recherche et d'information sociopolitiques, ${ }^{\circ} 1918$, p. 2-32.

Blogie, E. (2015). «En formation anti-radicalisation avec les imams belges ». Le Soir. 19 novembre.

Christians, L.-L., M. Magits, C. Sägesser et L. De Fleurquin. (2010). La réforme de la législation sur les cultes et les organisations philosophiques non confessionnelles. Rapport du groupe de travail instauré par arrêté royal du 13 mai 2009. Bruxelles, SPF Justice.

Conseil de l'Europe. (2008). Livre blanc sur le dialogue interculturel. Vivre ensemble dans l'égale dignité. Strasbourg, Conseil de l'Europe.

de Coorebyter, V. (2005). "Retour sur la naissance d'un système paradoxal». Dans J.-F. Husson (dir.), Le financement des cultes et de la laïcité : comparaison internationale et perspectives. Namur, Les Editions namuroises, p. 91-100.

Favret-Saada, J. (2007). Comment produire une crise mondiale avec douze petits dessins. Paris, Les Prairies ordinaires.

Haut conseil à l'intégration. (2000). L'islam dans la République. Paris, La Documentation française.

Hermon-Belot, R. et S. Fath. (2005). « La République ne reconnaît... aucun culte ». Archives de sciences sociales des religions, vol. 50, $\mathrm{n}^{\circ} 129$, p. 7-13.

Husson, J.-F. (2007). La formation des imams en Europe. État des lieux. Bruxelles, Fondation Roi Baudouin.

Husson, J.-F. (2012). «Le financement public de l'islam - instrument d'une politique publique? ». Dans B. Maréchal et F. El Asri (dir.), Islam belge au pluriel. Louvain-la-Neuve, Presses universitaires de Louvain, p. 241-258.

Husson, J.-F. (2014). «La libre-pensée, une religion comme une autre? Financement public et gestion de la pluralité religieuse et philosophique en Belgique». Dans A.-S. Lamine (dir.), Quand le religieux fait conflit. 
Désaccords, négociations ou arrangements. Rennes, Presses universitaires de Rennes, p. 47-60.

Husson, J.-F. (2017). «L'État favorise-t-il le dialogue interconvictionnel? ». Espace de libertés, $\mathrm{n}^{\circ} 460, \mathrm{p} .48-51$.

Husson, J.-F. (2018). «Attentes des pouvoirs publics en matière de formation des cadres musulmans. Réflexions sur la base du cas belge ». Dans F. Messner et M. Abou Ramadan (dir.), L'enseignement universitaire de la théologie musulmane. Paris, Le Cerf, p. 149-162.

Husson, J.-F. et C. Sägesser. (2002). «La reconnaissance et le financement de la laïcité (II)». Courrier hebdomadaire du Centre de recherche et d'information socio-politiques, $\mathrm{n}^{\circ} 1760$, p. 3-52.

Husson, J.-F. et J. Dury. (2006). Pour une formation des imams en Belgique. Points de référence en Belgique et en Europe. Bruxelles, Fondation Roi Baudouin.

Husson, J.-F. et J. Mandin. (2014). Étude de faisabilité en vue de la création d'un Institut public d'étude de l'islam (IPEI). Liège, CEDEM - Université de Liège.

Keunings, L. (2009). Des polices si tranquilles. Une histoire de l'appareil policier belge au XIX ${ }^{e}$ siècle. Louvain-la-Neuve, Presses universitaires de Louvain.

Lijphart, A. (1981). Conflict and Coexistence in Belgium: The Dynamics of a Culturally Divided Society. Berkeley, University of California.

Machelon, J.-P. (2006). Les relations des cultes avec les pouvoirs publics. Paris, La Documentation Française.

Milot, M., P. Portier et J.-P. Willaime. (2010). Pluralisme religieux et citoyenneté. Rennes, Presses universitaires de Rennes.

Portalis, F. (1845). Discours, rapports et travaux inédits sur le concordat de 1801. Paris, Joubert.

Rigaux, M.-F., F. Mortier, J. Drijkoningen, J.-F. Husson, K. Leus et N. Smets. (2006). Le financement par l'Etat fédéral des ministres des cultes et des délégués du Conseil central laïque. Rapport de la Commission des sages. Bruxelles, SPF Justice. 
RTBF. (2016). «Plan anti-radicalisation: 3,3 millions pour rémunérer 80 nouveaux imams », 18 février.

Sägesser, C. (2013). « Le temporel des cultes dans la Belgique du XIXème siècle : Législation, règlementation, jurisprudence et pratiques ». Thèse de doctorat, Bruxelles, Université Libre de Bruxelles, Département d'Histoire, Arts et Archéologie.

Sägesser, C. et C. Torrekens. (2008). «La représentation de l'islam ». Courrier hebdomadaire $d u$ Centre de recherche et d'information socio-politiques, $\mathrm{n}^{\circ}$ 1996-1997, p. 3-55.

Torfs, R. (2005). «Église, État et laïcité en Belgique. Remarques introductives ». Dans J.-F. Husson (dir.), Le financement des cultes et de la laïcité : comparaison internationale et perspectives. Namur, Les Editions namuroises, p. 15-21.

Torfs, R. (2009). «Church Financing-towards a European Model?». Dans B. Basdevant-Gaudemet et $\mathrm{S}$. Berlingo (dir.), Le financement des religions dans les pays de l'Union européenne. Leuven, Peeters, p. 343-350.

Torrekens, C. et I. Adam. (2015). Belgo-Marocains, Belgo-Turcs : (auto)portrait de nos concitoyens. Bruxelles, Fondation Roi Baudouin.

Willaime, J.-P. (2008). Le retour du religieux dans la sphère publique. Vers une laïcité de reconnaissance et de dialogue. Paris, Editions Olivétan.

Willaime, J.-P. (2009). «Les laïcités belge et française au défi de la laïcité européenne». Dans F. Foret (dir.), Politique et religion en France et en Belgique. Bruxelles, Éditions de l’Université de Bruxelles, p. 161-177.

Wynants, P. (1986). Autres cultes (1598-1985). Tome 1. France - Belgique - GrandDuché. Namur, Fondation internationale Meuse-Moselle. 